\title{
Percutaneous coronary intervention in unprotected left main stem disease: the state of play
}

\section{N Curzen}

$\mathrm{P}$ ercutaneous coronary intervention (PCI) to the left main coronary artery (LM) presents important technical challenges that are both lesion and site specific. Available technology has improved and so has our understanding of how to optimise short and long term outcome, but nevertheless intervention to this area demands special consideration. Critical analysis of the literature can only be performed in conjunction with clear awareness of the limitations of the studies. Factors include: paucity of randomised trials comparing stent with surgery for LM lesions; the heterogeneous nature of most reported patient populations, so that papers often include a mixture of lesion types (ostial, body or bifurcation) or high and low risk patients; a lack of consistency of PCI technique, so that many series report a mix of plain old balloon angioplasty (POBA)/ stent/debulking or combinations of these. Interpretation of existing literature is therefore necessarily complex and imprecise. Furthermore, as is common in the realm of PCI, the speed of change of both equipment and technique make much of what is "established" potentially irrelevant to "state of the art" practice: the impact of drug eluting stents (DES), newer bifurcation techniques, and concomitant pharmacological treatment being obvious examples. Nevertheless, the most recent guidelines on revascularisation from both the European Society of Cardiology and American Heart Association/American College of Cardiology still recommend coronary artery bypass graft surgery (CABG) for the majority of patients with unprotected LM disease. ${ }^{12}$ It is in this context that the available data on LM PCI must be reviewed.

\section{TECHNICAL FEASIBILITY}

The initial angiographic success rates for contemporary (that is, using stents) series of LM PCI are universally high. In elective series, reported procedural success is consistently above $90 \%$ and in the majority is close to $100 \%$, even in series including bifurcation disease. ${ }^{3}$ By contrast, patients presenting with acute coronary syndromes caused by important LM stenosis represent a higher risk group and it is not surprising that in-hospital outcome data are therefore much less favourable: the procedural success in the emergency group of the (original) ULTIMA (unprotected left main trunk intervention multi-center assessment) series, for example, being $75 \%$ with an in-hospital mortality of $69 \%{ }^{4}$

The technical feasibility of LM stenting is therefore well established, particularly in elective cases.

\section{CLINICAL DETERMINANTS OF SURVIVAL AND OUTCOME}

Given the fact that (until recently) conventional wisdom considered LM disease to be in the surgical domain, it is perhaps ironic that many interventionalists' early experience of PCI in LM is dominated by patients turned down for CABG on the basis of unacceptably high risk! Distillation of the available literature ${ }^{35}$ reveals a generally consistent pattern of clinical risk factors that are summarised in table 1 . These factors incorporate co-morbid conditions, such as respiratory failure, renal impairment etc, via the inclusion of the well established surgical scoring systems. It is one of the challenges of modern intervention that we design a scoring system incorporating factors specific to PCI in order to be able to standardise our assessment of risk and thereby accurately compare patient populations and outcome.

The review by Marco and Fajadet ${ }^{5}$ summarises the inhospital outcome of LM PCI patients as follows: "...it is a general agreement that patients considered as "low risk" are defined as less than 75 years old, with LVEF $>40 \%$, and reference vessel diameter $>3.6 \mathrm{~mm}$ have a satisfactory outcome, with an in hospital mortality rate ranging between $0-2 \%$. For patients at higher surgical risk or those who are deemed inoperable, the in hospital mortality rate is higher ranging between 6 and 13\%." For example, in the study by Kosuga et al, patients undergoing directional atherectomy for LM disease could be divided into three groups according to presentation: acute (including those with acute myocardial infarction) $(\mathrm{n}=14)$, emergency $(\mathrm{n}=10)$, and elective $(\mathrm{n}=83)$. The in-hospital mortality was higher in the acute $(35.7 \%)$ and emergency $(40.0 \%)$ groups than in the elective $(3.6 \%)(p<0.0001)$ group. $^{6}$

Longer term outcome is driven predominantly by mortality and target lesion revascularisation (TLR) or target vessel revascularisation (TVR). Predictably, the best outcome is achieved in patients with lower clinical risk. In the Kosuga et al paper described above, ${ }^{6}$ the five year survival was $50.0 \%$ and $48.2 \%$ in the acute and emergency groups but $75 \%$ in the elective patients $(p<0.05)$. In a series of 270 patients at low risk, the one and three year survival was $97.7 \%$ and $96.8 \%$, respectively, with major adverse cardiac event (MACE)-free survival of $81.9 \%$ and $77.7 \%$ at the same time points. ${ }^{7}$ By contrast, the one year mortality for the high risk group in ULTIMA was $24.2 \%{ }^{8}$ Similarly, Takagi et al reported outcome in 67 consecutive patients undergoing PCI to unprotected LMS and found the overall cardiac survival at three years to be $91 \%$ (although there were three other non-cardiac deaths). ${ }^{9}$ The subgroup of patients with a Parsonnet score of $>15$, however, had a three year mortality of $21.4 \%$ versus only $4.2 \%$ in those in whom the score was $<15(p<0.02)$. The overall angiographic restenosis rate was $31.4 \%$ (in the $85 \%$ of the original population who were eligible). It is clear, therefore, that it is possible to achieve excellent survival rates in these patients and that these are modified in a predictable fashion by the initial risk profile of the patient.

\footnotetext{
Abbreviations: $C A B G$, coronary artery bypass graft surgery; DCA, directional coronary atherectomy; DES, drug eluting stents; IVUS, intravascular ultrasound; $L M$, left main coronary artery; $M A C E$, major adverse cardiac event; $M L D$, minimal lumen diameter; $\mathrm{PCl}$, percutaneous coronary intervention; POBA, plain old balloon angioplasty; RESEARCH, rapamycin-eluting stent evaluated at Rotterdam cardiology hospital; SYNTAX, Synergy with Taxus; TLR, target lesion revascularisation; TVR, target vessel revascularisation; ULTIMA, unprotected left main trunk intervention multi-center assessment
} 
Table 1 Clinical markers of high risk in unprotected LM coronary $\mathrm{PCl}$

Clinical

Age $>75$ years

Diabetes

Renal failure

High Parsonnet or EuroSCORE

Acute myocardial infarction or cardiogenic shock

Emergency presentation

Angiographic

Distal/bifurcational location

LV dysfunction (LV ejection fraction $<40 \%$ )

Multiple dilatations

Post-PCI MLD of $<3.6 \mathrm{~mm}$

Post-PCI MLA of $<7-8 \mathrm{~mm}^{2}$

LV, left ventricular; MLA, minimal lumen area; MLD, minimal lumen diameter; $\mathrm{PCl}$, percutaneous coronary intervention.

Characteristically, however, the restenosis rates in such series usually lie in the range $20-40 \%$, a factor that may be important if restenosis contributes to the sudden death rate in such patients.

\section{ANGIOGRAPHIC DETERMINANTS OF OUTCOME}

The reference diameter of the LM and the length of the lesion ${ }^{10}$ are reported to be determinants for risk of restenosis, as would be expected from the vast amount of data to that effect in relation to all other PCI. Thus, in both this and a separate series, ${ }^{7}$ reference minimal lumen diameter (MLD) of $<3.6 \mathrm{~mm}$ was an independent risk factor for restenosis. In the latter study of 270 patients, binary in-stent restenosis at angiographic follow up was $21.1 \%$ with reference vessel size being the only predictor of restenosis after multivariate analysis.

A further angiographic factor that determines outcome in LM PCI is the site of the stenosis. The immediate and long term outcome of stenting the ostium and body of the LM appears to be highly favourable. A recent retrospective observational study reported the outcome in 71 patients who underwent nonbifurcational LM PCI. ${ }^{11}$ Despite the fact that the overall stent rate was only $64 \%$, the total one year survival was $98.6 \%$ with a subsequent annual mortality of $2.5 \%$. By contrast, in a different paper the restenosis rate was significantly higher in the patients treated with distal LM stenoses than in those with ostial or body lesions. ${ }^{10}$ This observation has been reproduced by others. Mulvihill et al found freedom from TLR at a mean (SD) of 36 (20) months of $92 \%$ for non-distal and $73 \%$ for distal $(p=0.003)$ stenoses. ${ }^{12}$ It is important to note that as well as the expected higher rate of TLR $(26 \% v 8 \%)$ in the distal stenosis group, the mortality in these patients was also higher $(31 \%$ v $14 \%, \mathrm{p}=0.01)$.

\section{INTRAVASCULAR ULTRASOUND DETERMINANTS OF OUTCOME}

The discrepancy between lesion assessment by angiography as compared to intravascular ultrasound (IVUS) is seldom better demonstrated than in LM disease (fig 1). IVUS allows an accurate assessment of the reference vessel size, extent of atheroma burden, composition of the lesion (particularly extent of calcification) and lesion length: all factors that are frequently underestimated by angiography alone. Further, IVUS allows a critical assessment of the success of stent placement and deployment in a more thorough manner than is achievable by angiography. For these reasons, many interventionalists believe that IVUS assessment is a mandatory component of LM PCI. Despite this, the published data are conflicting on the value of IVUS, and this is partly because there is a surprising paucity of studies looking in a systematic way at IVUS assessment of the LM and its impact on subsequent PCI. There are data to suggest that IVUS guidance of LM stenting results in more aggressive stent deployment in terms of post-dilatation at higher pressure or using bigger balloons. ${ }^{13}$ Furthermore, in a series of 87 cases (although in this case the LM lesions were protected), the IVUS derived post-PCI minimal luminal area (MLA) was the sole independent predictor of TVR: when the MLA was $<7 \mathrm{~mm}^{2}$, TVR was $50 \%$, and when $>7 \mathrm{~mm}^{2}$ TVR was $7 \%{ }^{14}$ Surprisingly, perhaps, a more recent series does not apparently find the same (intuitive) relation between bigger stent result and lower TVR. ${ }^{15}$ In this paper the post-PCI MLD was indeed significantly larger in the IVUS guided stent group, but the angiographic restenosis rates were similar at six months $(18.6 \% \vee 19.5 \%$; $=0.5)$. This is difficult to explain although comparison between the studies is made difficult by important differences in PCI technique, the second study concentrating upon debulking.

Further data are undoubtedly required to address the question: if IVUS were employed routinely for assessment of all LM lesions before a procedure, in how many cases would it influence the PCI strategy?

\section{DRUG ELUTING STENTS IN LM PCI}

There are currently only limited data available on the effect of DES in LM PCI. In one series of 43 patients with a variety of LM lesions treated with Cypher stents, the TVR rate was 23\% overall, but $71 \%$ in the (small) diabetic subgroup. ${ }^{16}$ There were also a limited number of LM lesions ( 17 out of 508, 3\%) included in the RESEARCH (rapamycin-eluting stent evaluated at Rotterdam cardiology hospital) registry in whom there were no TVR events, ${ }^{17}$ but more data are needed. In the meantime, it is an important practical limitation, given the MLD data derived from IVUS studies, that interventionalists in the UK do not have access to DES of $>3.5 \mathrm{~mm}$ diameter.

\section{DEBULKING: DOES IT IMPROVE OUTCOME?}

The literature on LM PCI contains many series that include some patients who had either rotational atherectomy or directional coronary atherectomy (DCA). It is not clear, however, why these patients were chosen and is further complicated by the fact that the patients were then treated in a variety of other ways by balloon or stent or neither. Debulking can unequivocally be said to be feasible, but there are few data to suggest that it improves outcome in terms of either mortality or TVR/TLR. There are two immediate questions in relation to debulking in LM lesions. (1) Does the ability to reliably debulk heavily calcified plaques allow more LM lesions to be accessible to stenting? (2) Does debulking allow for superior stent deployment and improve outcome by optimising lesion preparation?

\section{CONCLUSION}

The frequency of unprotected LM PCI is increasing and the clinical outcome of this activity is apparently improving, although TVR/TLR rates in series without DES remain higher than we have now come to expect in other coronary territories. There remains appropriate concern about the inferior outcome of distal LM PCI involving the bifurcation compared to lesions in the ostium or body. It is now especially important to compare, by randomised study, the outcome of LM stenting with CABG, looking at that the bifurcation subgroup in particular detail. The SYNTAX (Synergy with Taxus) trial is currently being designed with this question in mind. New advances, specifically DES, are likely to improve outcome, because in many series of elective LM PCI mortality is so low that the only driver for MACE remains restenosis. It is interesting that even with a post-PCI MLD of over $4 \mathrm{~mm}$, restenosis rates in these lesions treated with bare metal stents remain nearly $20 \%$. This implies that 

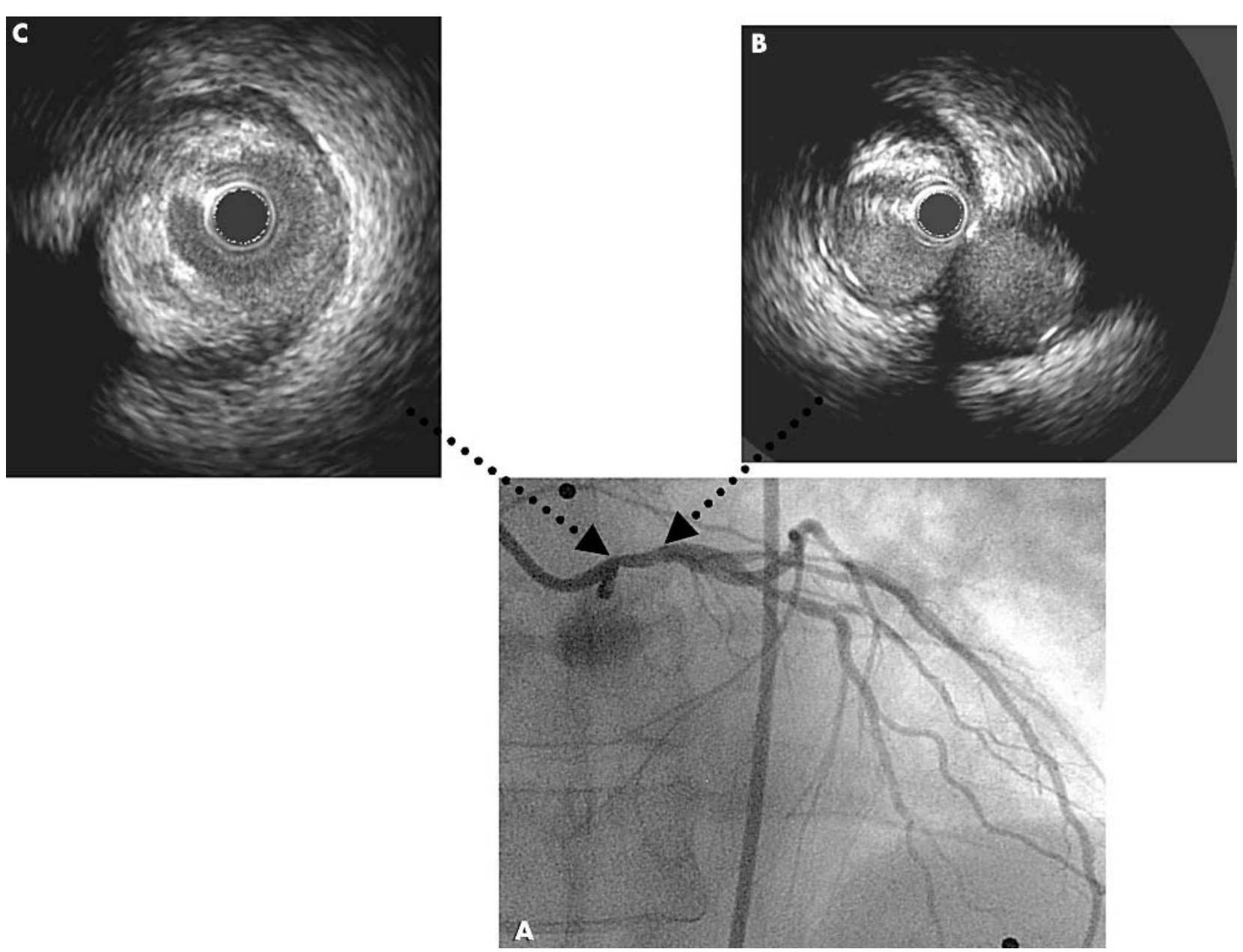

Figure 1 The discrepancy between angiography and intravascular ultrasound (IVUS) in unprotected left main coronary artery (LM) disease. This 50 year old man was admitted with a troponin positive acute coronary syndrome. Angiography in his local hospital demonstrated an important stenosis in the proximal segment of his left main coronary that caused pressure damping upon engagement (panel A). His remaining coronary tree was unobstructed and he was referred for percutaneous coronary intervention. IVUS showed much more extensive atheroma throughout his $L M$, including the distal segment and bifurcation, where the area stenosis was just over $50 \%$ (panel B). Furthermore, on IVUS the reference vessel diameter in the LM was $3.9 \mathrm{~mm}$ (panel C), although on angiography we estimated using a stent of 2.75-3.0 mm. Pictures courtesy of Dr H Gray and N Curzen.

the LM may, for some reason, be more susceptible to restenosis than other parts of the coronary tree. The challenge will be to deploy DES of the relevant diameter to such lesions. Finally, there remains a question mark over the clinical outcome of the diabetic subgroup undergoing LM PCI. There is little doubt, however, that as the answers to these key questions become available, LM PCI will steadily become the treatment of choice for the majority of these lesions.

Correspondence to: Dr N Curzen, Wessex Cardiothoracic Unit, Southampton General Hospital, Tremona Road, Southampton S016 6YD, UK; nick.curzen@suht.swest.nhs.uk

\section{REFERENCES}

1 Silber $S$, et al. European guidelines for percutaneous coronary intervention (PCI). Eur Heart J (in press).

2 American Heart Association, American College of Cardiology. AHA/ACC guideline for PCl: executive summary. Circulation 2001;103:3019-41.

3 Balachandran $\mathrm{K}$, Oldroyd $\mathrm{K}$. PCl in unprotected left main coronary disease. Br J Cardiology (Acute \& Interventional Cardiology) 2003;10:AIC22-7.

4 Ellis S, Tamai H, Nobuyoshi M, et al. Contemporary percutaneous treatment of unprotected left main coronary stenosis; initial results from a multicentre registry analysis 1994-1996. Circulation 1997;96:3967-72.

5 Marco J, Fajadet J. Unprotected left main stenting. The Paris Course on Revascularisation, 2004:171-180.

6 Kosuga K, Tamai $\mathrm{H}$, Ueda K, et al. Initial and long term results of directional coronary atherectomy in unprotected left main coronary artery. Am J Cardiol $2001 ; 87: 838-43$
7 Park S, Park S, Hong M, et al. Long-term (3-year) outcomes after stenting of unprotected $L M$ coronary artery stenosis in patients with normal left ventricular function. Am J Cardiol 2003;91:12-16.

8 Tan WA, Tamai H, Park S, et al. Long-term clinical outcomes after unprotected left main trunk revascularisation in 279 patients. Circulation 2001;104:1609-14.

9 Takagi T, Stankovic G, Finci L, et al. Results and long-term predictors of adverse clinical events after elective $\mathrm{PCls}$ on unprotected left main coronary artery. Circulation 2002;106:698-702.

10 De Lezo JS, Medina A, Romero M, et al. Predictors of restenosis following unprotected left main coronary stenting. Am J Cardiol 2001;88:308-10.

11 Brueren B, Ernst J, ten Berg J, et al. Long term follow up after elective percutaneous coronary intervention for unprotected non-bifurcational left main stenosis: is it time to change the guidelines? Heart 2003;89:1336-39.

12 Mulvihill N, Boccalatte M, Farah B, et al. Anatomical location of lesion predicts restenosis in unprotected left main coronary stenting. Eur Heart $J$ 2002;4:suppl 309

13 Hong $M$, Park S, Lee $C$, et al. Intravascular ultrasound findings in stenting of unprotected left main coronary artery stenosis. Am J Cardiol 1998;82:670-3.

14 Hong M, Mintz G, Hong M, et al. Intravascular ultrasound predictors of target lesion revascularisation after stenting of protected left main coronary artery stenoses. Am J Cardiol 1998:83:175-9.

15 Park S, Hong MK, Lee C, et al. Elective stenting of unprotected left main coronary stenosis. Effect of debulking before stenting and IVUS guidance. J Am Coll Cardiol 2001;38:1054-60.

16 Cheiffo A, Orlic D, Airoldi F, et al. Early and mid-term results of Cypher stents in unprotected left main. J Am Coll Cardiol. 2004;43: 21 A-1116-8 (abstract)).

17 Lemos P, Serruys P, van Domburg RT, et al. Unrestricted utilisation of sirolimus-eluting stents compared with conventional bare stent implantation in the "real world": the rapamycin-eluting stent evaluated at Rotterdam Cardiology Hospital (RESEARCH) registry. Circulation 2004;109:190-5. 